\title{
Educational Laboratory Complex for the Study of Complicated Systems
}

\author{
Sergey I. Suyatinov ${ }^{1 *}$ \\ ${ }^{1}$ Bauman Moscow State Technical University, 2nd Baumanskaya str., 5/1, 105005, Moscow, Russia
}

\begin{abstract}
Laboratory practice is an important part of the educational process in a technical university. It helps to develop the skills of independent work of a future engineer and to consolidate theoretical knowledge. Due to a change-over to the digital economy, the introduction of complex production systems and their digital twins, the organization of laboratory work corresponding to the current state of the art gets more complicated. The article presents a biotechnological educational research complex for laboratory practice of the future specialists in the field of managing complex organizational and technical systems. Main feature of the complex is that the student performs two functions at the same time. $\mathrm{He}$ is both a researcher and an object of study, representing a complex system. The initial information in the process of laboratory work is the biosignals registered by mini sensors. The main purpose is to identify and evaluate the functional state of a person as a complex system. Various methods and algorithms for processing time series are used. Being an effective form of active learning, this approach motivates the student not only to acquire knowledge, but also to actively search for it. We give an example of using the complex to create a digital twin.
\end{abstract}

\section{Introduction}

The purposes of identification and management of complex systems arise when solving problems in various fields: technical, biomedical, economic, social. Therefore, we have a problem to determine a universal approach to the description and study of such systems. The results of synergetic studies showed that at a certain level of abstraction, there are deep analogies between the behavior of various complex systems [1-4]. It determines the universality of the approach to the complex systems study.

Therefore, from the perspective of the modern development of science, the best solutions to the problems of studying complex systems lie in the interdisciplinary field. The proof is the worldwide recognition of the strategy of scientific and technological development based on nano-, bio-, information and cognitive (NBIC) technologies [5, 6]. In particular, bioinformation technologies are a convenient tool for studying complex systems of various origins.

Bioinformation technologies in this case are based on the well-known feature of complex systems [7]. In particular, the more complex the system, the stronger the similarity

*Corresponding author: $\underline{\text { ssi@bmstu.ru }}$ 
between the features of its own and human behavior. Therefore, by exploring and revealing the patterns of human functioning and subsystems of his body, you can get an idea of the fundamental properties of complex systems and methods to describe them. This fact underlies the anthropomorphic approach to the study of complex (including industrial) systems.

Modern complex production is characterized by significant uncertainty. Its condition can be identified, as a rule, only by the results of indirect measurements and observations during monitoring. A feature of modern monitoring systems for complex objects is the large amount of heterogeneous data received. Therefore, a relevant task is to develop the theory and practice of collecting and joint processing of large volumes of heterogeneous data in order to identify and assess the state of a complex system [8].

Considering fast pace of development of scientific and technological progress, the steady complication of technology, we need to improve the engineering preparation process. This is primarily about the introduction of information technology, as well as active, gaming forms of organization of the educational process [9-11].

The future engineer should get acquainted with the behavior peculiarities and description of complex systems well back when being a student. At the same, the training process should include research work using real equipment.

Laboratory work has always been and is an integral part of the training of an engineer. However, with the complication and appreciation of technical devices, it has become problematic to equip laboratories with the required devices in their natural form.

An alternative option is based on the use of mathematical models of real systems objects. In the case of complex systems with the structural-parametric uncertainty, this option needs serious modification. In addition, the verification of the proposed options is preferably carried out on real systems, which, however, is not always possible.

When organizing laboratory and practical work, many problems associated with the study of complex systems are solved if the anthropological approach is used. The training and laboratory complex under consideration allows you to clearly demonstrate specific problems and possible methods for solving them with concrete examples.

\section{Laboratory Practice: Tasks and Objects of Research}

The innovative nature of the development of modern production, a change-over to the digital economy impose new requirements for the organization of the educational process and, in particular, for the organization of laboratory work. Summarizing the results [12-14], we can thus determine the tasks of training in the laboratories of future engineers at the present stage:

- In the laboratory, a student learns to experiment;

- The laboratory should be a place where a student studies new, completely unknown objects and phenomena;

- In the laboratory, a student not only consolidates theoretical knowledge, but also learns to independently acquire new ones, realizing the principle «learning through research»;

- Laboratory courses should be based on natural science and build a student's understanding of the real world;

- Laboratory work stimulates creativity;

- The training laboratory is the place where a student is attracted to engineering.

Laboratory work is especially important for disciplines, the objects of study of which are characterized by a high level of abstraction and mathematization. They allow students to see the physical principles of the functioning of real devices behind abstract formulas. 
For example, a simple electromechanical system clearly demonstrates the main effects of automatic control described in a theoretical course.

However, such works are relevant in the case of a certain (deterministic) and unambiguous description of laboratory setup models that are characteristic of classical objects.

In the case of studying complex systems with their "innate" uncertainty, the theory operates with such concepts as synergetics, research modeling, multiscale models, information fusion, basic models, reconstruction, entropy, etc.

A practical demonstration of these concepts is very difficult. To a large extent, this is associated with the lack of an object of study - a complex system that operates in a training laboratory.

At the same time, the student himself (his body) presents a complex system of natural origin.

It should be noted here that in modern technical, physical, economic and other sciences, the systems considered are so complex that they resemble biological systems in their behavior [7]. Therefore, using the example of research and identification of the state of the human body, it is possible to study some fundamental principles of the functioning of complex systems of various nature.

\section{Features of the Biotechnological Complex for the Study of Complicated Systems}

In the considering complex, an elaborate object of research is a human, his functional state (FS). Until recently, the study of FS was possible only in special laboratories using bulky and expensive hardware.

Improvement of microelectronic devices and, first of all, sensory and computer equipment allows creating safe compact information and computing systems for assessing the FS of a human operator without violating its normal mode of operation $[15,16]$. Similar systems were selected as a prototype of the biotechnological complex under consideration.

It should be especially noted that the student in this case plays a dual role, being both a researcher and an object of study.

Thus, the student determines his functional state (its transformation pattern), under the influence of disturbing factors, using the latest achievements in the field of signal processing, identification, pattern recognition.

Fig. 1 schematically represents the summarized idea of building a biotechnological complex. It is easy to notice that this is a typical scheme for monitoring the state of a real object of any complexity. The complexity of the object will determine the choice of appropriate methods for processing time series. It should be noted that the methods and techniques for transforming information at each stage are studied by students at the corresponding courses. 


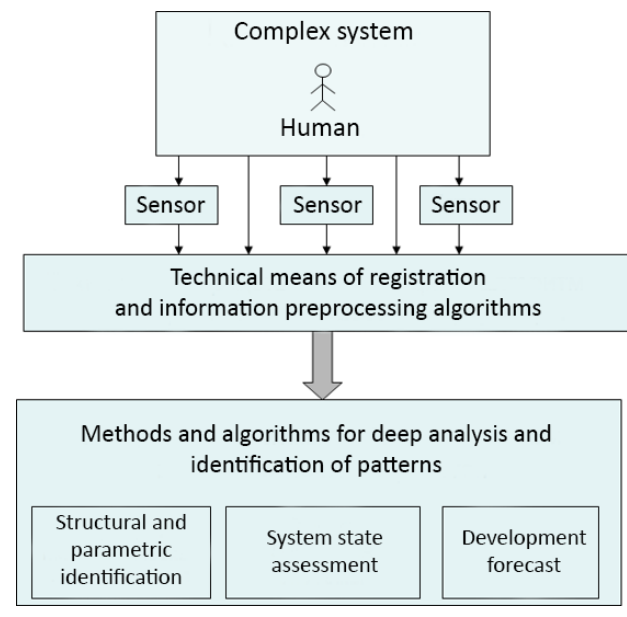

Fig. 1. Functional diagram of the complex.

The initial information for assessing the state is the time series of biosignals of various origin. In this case, the assessment of human FS is carried out using cardiac cycle biosignals (ECG and sphygmogram).

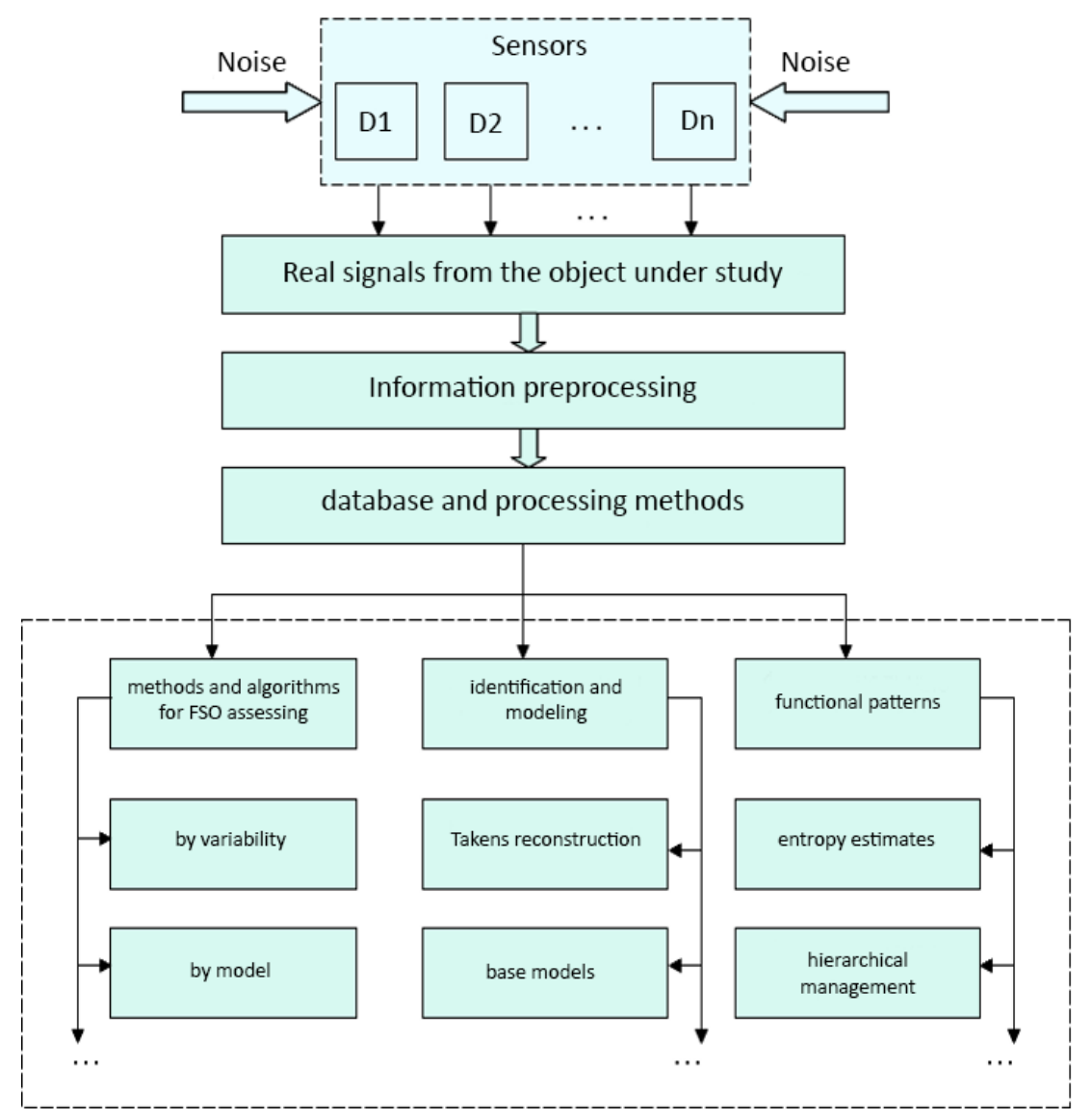

Fig. 2. An example of directions and methods of using biosignals in the form of time series for assessing human FS. 
Fig. 2 shows some areas and methods of using biosignals in the form of time series for assessing human FS, diagnostics, and studying the fundamental principles of organization and management in complex systems.

Various options for the technical implementation of the biotechnological complex are possible. If we focus on imported components, the complex implemented using equipment from National Instruments will have the greatest functionality.

The complex was created at the department «Automatic control systems» of BMSTU. Unified functional blocks allow for quick reconfiguration for a specific task, and the methods of graphic programming of the LabVIEW software complex, together with a rich library of data processing and visualization programs, greatly facilitate the research part.

Figure 3 shows a photo of Plessey Semiconductors EPIC sensors for ECG recording [17].

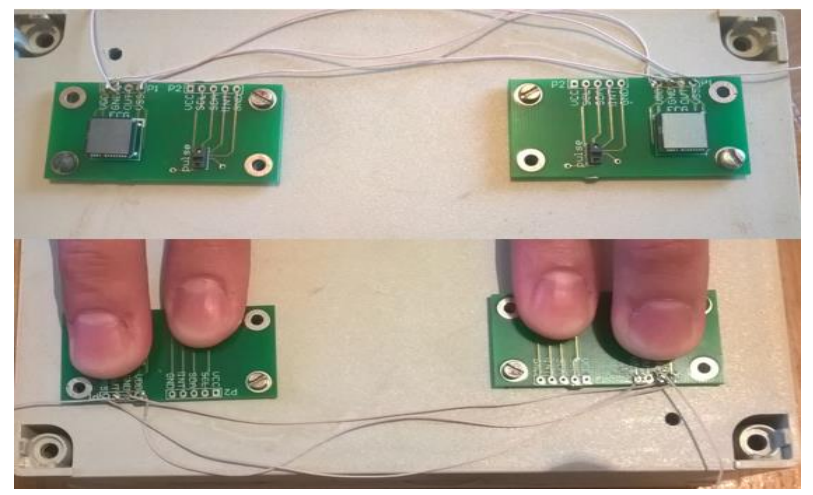

Fig. 3. The sensors (above) and the biosignals reading (below).

To read one ECG lead, we need two identical sensors connected by a differential circuit. Sphygmogram is recorded using the MAX30100 pulse wave sensor.

Work with real equipment will provide further rapid adaptation of the graduate in the workplace, which is a very important task of engineering education [18, 19].

\section{Examples of Complex Use in the Study of Complicated Systems}

The complex under consideration allows us to solve various typical educational research problems that arise when monitoring real complex systems. The range of these tasks is wide enough, starting with filtering and digital processing algorithms for real noisy signals and ending with creation of hierarchical digital twins [20-22]. Moreover, one of the most important monitoring tasks is to assess the state of a complex system. Let's take a closer look at the following two examples.

\subsection{Digital twin is a computer image of a complex system}

A distinctive feature of the digital economy is the organization of production in the form of a cyber-physical system. Fundamentally, any cyber-physical system includes two information-related components: a physical object and its digital twin [23-25].

A digital twin (DT) provides a mathematical basis for the determination of numerical data characterizing the functional state of the system. 
From the point of view of technical implementation, a digital twin is a structured set of data and algorithms that allows algorithmically simulating the state and behavior of the system and its elements under various external and internal influences.

Fig. 4 presents a generalized structure of the system «production - digital twin».

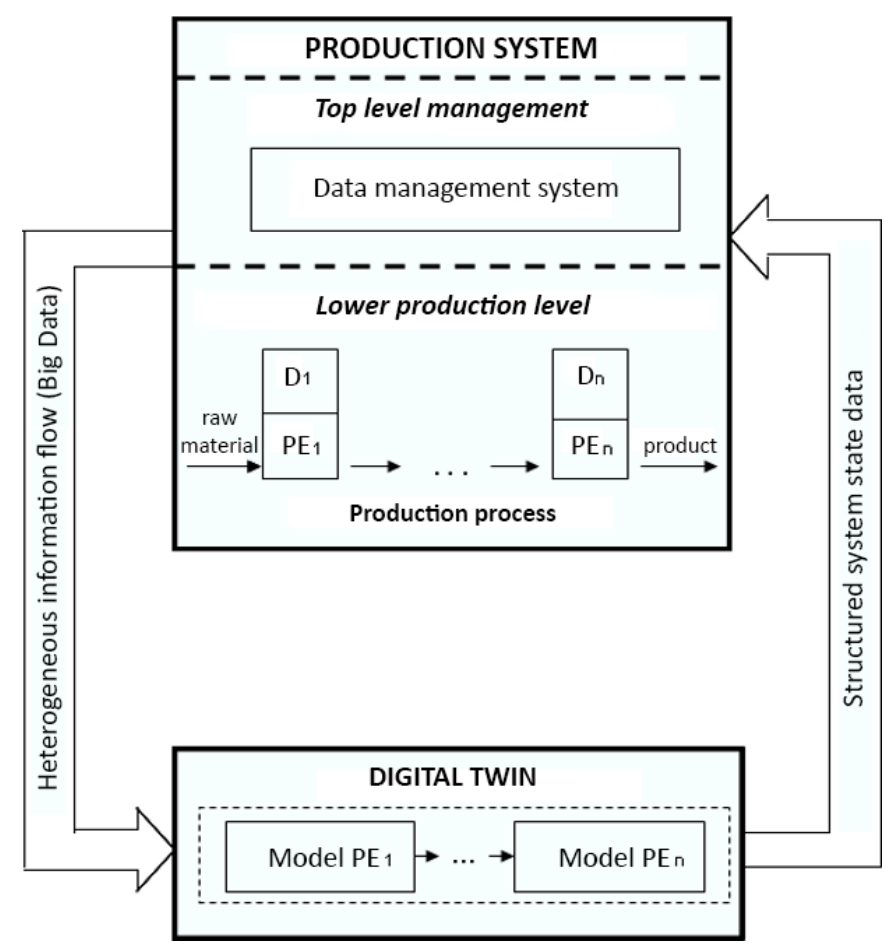

Fig. 4. Production system and its digital twin.

At the production level with the help of sensors $\mathrm{D}_{1}, \ldots, \mathrm{D}_{n}$ measuring information is collected that characterizes the state of working processes and production elements $\mathrm{PE}_{1}, \ldots, \mathrm{PE}_{\mathrm{n}}$. This information is used both for building models of PE functioning and for further assessment of the correspondence of current characteristics of work processes and their model implementations.

Here, the digital twin is represented by a set of mathematical models of the functioning of production elements of $\mathrm{PE}_{\mathrm{i}}$. Digital twin brings many benefits to cyberphysical systems. For example, with its use, the problem of assessing the functional state of equipment is solved. This allows you to switch from scheduled repairs to repairs in actual condition.

As an example of constructing a simple digital twin of a complex system, we consider the solution to the problem of structural and parametric identification of the cardiovascular system. 
Fig. 5 shows a simplified version of the structure «cardiovascular system - digital twin». Considering the nature of the real object, such a structure is more correctly to be called «cyber-biological system».

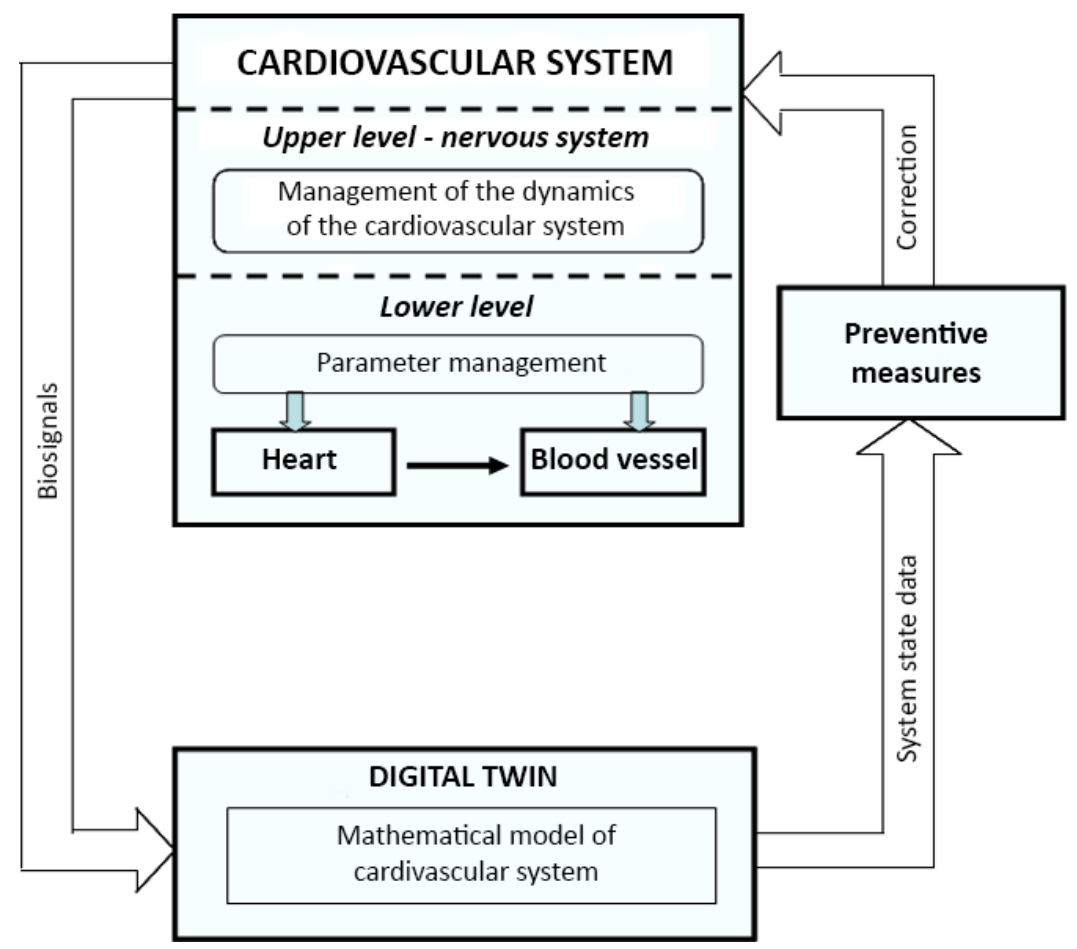

Fig. 5. The structure of the cyber-biological system.

The structural analogy with the cyber-physical production system is obvious. The difference lies in the fact that in the production system information on the state of production is received and used by management personnel located inside the production system. In the second case, the decision maker is outside the system under study.

It should be noted that, despite the transparency of the ideas for creating a DT, their practical implementation is a complex scientific and technical problem. This is primarily due to the need to structure large volumes of heterogeneous information and build fairly simple models of complex systems (identification problem).

A possible solution to the identification problem is based on the concept of basic mathematical models. Such models describe key processes in complex systems at a given spatial and temporal scale.

The oscillatory processes of the cardiocycle are crucial in this case. From the theory of oscillations, several basic differential equations are known that describe oscillatory processes. Therefore, in accordance with the concept of basic mathematical models, we describe the dynamics of the vascular wall by the van der Pol - Rayleigh equation:

$$
\ddot{x}+\left[\varepsilon_{1}\left(x^{2}-r^{2}\right)+\varepsilon_{2}\left(\dot{x}^{2}-\omega_{0}^{2} \cdot r^{2}\right)\right] \cdot \dot{x}+a x=P\left(\omega_{0} t\right) .
$$

Here $x$ is the movement of the vascular wall recorded by the sensor; $P\left(\omega_{0} t\right)$ - pressure on the vascular walls caused by cardiac activity. The advantage of the presented model is that the parameter $a$ has physical meaning: it allows of blood vessels due to the work of smooth muscles enveloping them. 
The choice of this particular differential equation is based on the results of previous studies $[20,22,26]$. In a similar way, a model of the pumping function of the heart muscle can be compiled using, for example, the Hill model.

Another option is the Volterra artificial neural network model. The input of the network is the ECG signal, and the output is the pressure signal $P\left(\omega_{0} t\right)$, specified as SCG. The obtained simplified model of the cardiovascular system in the form of a serial connection of the heart model and the model of vessel movement is presented in Fig. 6.

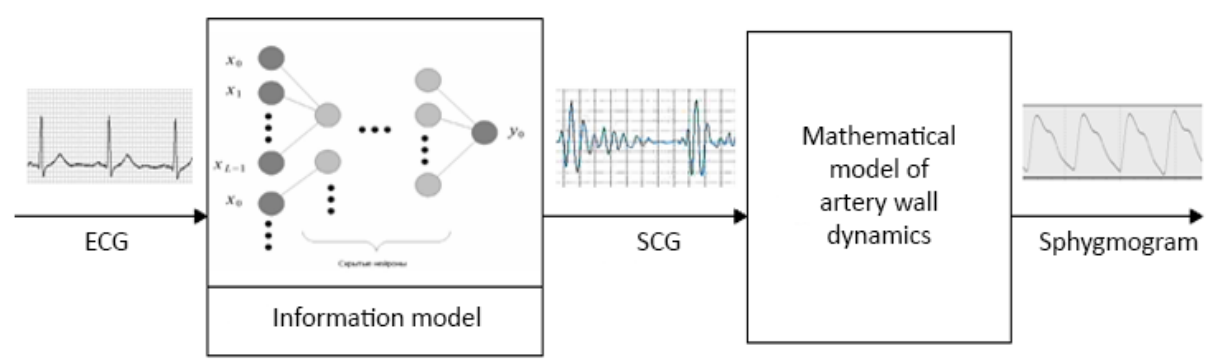

Fig. 6. Cardiovascular system model.

Thus, structural identification is implemented. Parametric identification is carried out using an ECG signal at the system input and a sphygmogram signal at the output. The obtained model parameters are integral indicators of the state of the cardiovascular system.

It should be emphasized that the described example is an option of the construction of a digital twin of such a complex object as the cardiovascular system. In practice, such a model can be an integral part of the representation of the digital twin of a human operator [26].

The research part of the work may include various tasks related to the choice of the model structure, methods for identifying parameters and clustering data (weighting factors).

\subsection{Entropy methods for assessing the state of a complex system}

Methods based on the calculation and analysis of information entropy are widely used in assessing the state of a complex system [27-29]. Evaluation of entropy allows to assess the complexity of the production system, the level of organization and stability of work, the state of workability of the human operator.

Let us consider an example of the assessment of entropy and its use in assessing the state of the cardiovascular system. The source data is the time series of the registered biosignals of the cardiocycle. These can be ECG signals, photo- or plethysmograms. Preprocessing the selected signal includes filtering and trend removal.

We specify them by $p_{i}$ as the probability of the system being in state $i$, which is characterized by a set of parameters (state vector) $Q_{i}$. All functional states form a complete system of events. In this case, the relation looks as $\sum_{i=1}^{n} p_{i}-1$.

By the uncertainty (entropy) of discrete quantities $p_{i}$ we understand the following criterion:

$$
H=-\sum_{i=1}^{n} p_{i} \log p_{i}
$$


If, for example, the system is increasingly in the $l^{\text {th }}$ state, then the probability $p_{l}$ tends to 1. In the limiting case when $p_{l}=1$, entropy is zero and the system becomes deterministic. If the system is indifferent to its states, then the probabilities are equal to each other, and the uncertainty calculated by the formulas acquires the maximum value. Thus, the uncertainty of the system lies within $0 \leq H \leq H_{\max }$, where $H_{\max }$ - some maximum value of entropy for a given system. The greater the entropy, the more the system is unpredictable.

The above formula (2) is a classic description of information entropy. However, for various reasons, the use of this formula is not possible in the analysis of real biological systems. Therefore, a number of estimating entropies were developed, which make it possible to get accurate information on the state of the system and on the degree of its ordering.

Pincus [30] proposed a family of statistics called Estimating Entropy (ApEn), to evaluate signal regularity, i.e. the existence of identical patterns in a time series. The specific algorithm for calculating the approximate entropy $\operatorname{ApEn}(m, r, N)$ is characterized by three parameters:

$m$ - determines the size of the time window containing $m$ values of a series;

$r$ - sets the tolerance for the characteristic of similarity (regularity) of vectors $Q_{i}$;

$N-$ this is the length (dimension) of the original time series.

Let us have a time series of measurements, for example, electric potentials of the heart (ECG) of dimension $N$, where $q(i)$ - is an $i^{\text {th }}$ value of the series $Q$. We choose a time window containing $m$ values of the series and present the original series as a set of vectors $Q(i)$ as follows:

$$
\begin{aligned}
& Q(1)=[q(1), q(2), \ldots, q(m)] \\
& Q(2)=[q(2), q(3), \ldots, q(m+1)], \\
& Q(3)=[q(3), q(4), \ldots, q(m+2)], \\
& \ldots \\
& Q(N-m+1)=[q(2), q(3), \ldots, q(m+1)] .
\end{aligned}
$$

The time series thus obtained are used to calculate the similarity coefficient $C_{i}^{m}(r)$. Here $r$ defines the tolerance for the characteristic of similarity (regularity). The similarity coefficient $C_{i}^{m}(r)$ is measured within a given tolerance of the regularity of time series similar to this sample, presented in a time window of length $m$. To do this, you can use various proximity measures and methods for determining similarity, for example, the least squares method.

For the given values $1 \leq i \leq N-m+1$ the coefficient $C_{i}^{m}(r)$ is determined as follows:

$$
C_{i}^{m}(r)=\frac{K}{N-m+1}
$$

Here $K$ is a number of vectors $Q(j)$, remote from a given vector $Q(i)$ no more than for a distance $r$. The algorithm for calculating the estimating entropy is as follows.

1. Split the initial time series into $N-m+1$ segments by $m$ measurements in the segment;

2. Select a vector $Q(i)$ in an $i$ cycle of the exhaustive search method;

3. Calculate the distance $L_{i}^{k}$, equal to the scalar product of the vector $Q(i)$ by $Q(k)$, where $k \in[1,(N-m)]$;

4. Check the condition $L_{i}^{k} \leq r$. The number of vectors satisfying this condition will determine the value $K$. 
5. Then calculate by the formula (3) the $C_{i}^{m}(r)$;

6. Calculate $F^{m}(r)=\frac{\sum_{i=1}^{N-m+1} \log \left[C_{i}^{m}(r)\right]}{N-m+1}$, where $F^{m}(r)$ - average value of the logarithm of $C_{i}^{m}(r)$;

7. Increase the window size by 1 and perform all the previous steps. As a result, we get the value $F^{m+1}(r)$.

The final approximate value of entropy is calculated by the formula:

$$
\operatorname{ApEn}(\mathrm{m}, \mathrm{r}, \mathrm{N})=F^{m}(r)-F^{m+1}(r)
$$

Approximate entropy estimates the logarithm of the probability that time series that are close to $m$ observations remain close and in the subsequently. The greater the regularity (i.e., the greater the likelihood of similar patterns), the less the entropy. Therefore, the entropy is a good tool for assessing the regularity of a time series, and, therefore, a criterion for assessing a human functional state.

Thus, the algorithm for assessing the organization (regularity) of the system includes the following steps:

1. Biosignal registration;

2. Time series formation and initial processing;

3. Estimation of the regularity of a time series based on the calculation of an approximate entropy;

4.Comparison of the obtained estimates of the entropy with the control entropy indicators of regularity;

5. Interpretation of results from the standpoint of assessing the functional state.

The research part of the work may include tasks related to the selection of optimal values of the parameters $m, r$ and $N$, control entropy indicators, as well as the solution of the problems of clustering and classification of the obtained entropy values for various functional states.

\section{Conclusion}

We presented a training and laboratory complex, intended for the development of methods and algorithms for processing time series in order to assess the state of complex systems.

A feature of the complex is that the student plays two roles: the researcher and the object of study. Study of the body with the help of the studied methods is a successful form of active learning.

In general, the capabilities of the presented complex allow us to solve the problems of modern engineering education in the era of the digital economy and to strengthen the role of the interdisciplinary focus of design work, developing creative activity, independence in the scientific and technical search, forming the need for constant updating of knowledge.

The specific capabilities of the complex are shown in the examples of constructing a digital twin of the cardiovascular system and the entropy assessment of the degree of organization of a complex biosystem. 


\section{References}

1. H. Haken \& J. Portugali, Information and Self-Organization, Entropy, 19(1), p. 18 (2017). doi:10.3390/e19010018

2. F. Capra, The systems view of life a unifying conception of mind, matter, and life, Cosmos and History, Vol. 11, I. 2, pp. 242-249 (2015)

3. G. Malinetskii, S. Manenkov, N. Mitin \& V. Shishov, A cognitive challenge and information technologies, Herald of the Russian Academy of Sciences, 81(4) (2011). DOI: $10.1134 / \mathrm{S} 1019331611040034$.

4. V.E. Karpov, V.B. Tarassov, Synergetic Artificial Intelligence and Social Robotics, Abraham A., Kovalev S., Tarassov V., Snasel V., Vasileva M., Sukhanov A. (eds) Proceedings of the Second International Scientific Conference "Intelligent Information Technologies for Industry" (IITI'17), IITI 2017, Intelligent Systems and Computing, Vol. 679, Springer, Cham (2018)

5. E.G. Kamensky, Context of NBIC-technologies development: Institutions, ideology and social myths, Mediterranean Journal of Social Sciences, Vol. 6, No. 6, pp. 181-185 (2015)

6. Converging technologies for improving human performance: Nanotechnology, biotechnology, information technology and cognitive science, Ed. by M.C. Roco and W.S. Bainbridge, The Netherlands: Kluwer Academic Publishers (2003)

7. H. Haken, Information and Self-Organization: A Macroscopic Approach to Complex Systems, Springer-Verlag Berlin Heidelberg, p. 258 (2006). DOI: 10.1007/3-54033023-2

8. D. Novikov, Cybernetics: from Past to Future, Berlin, Springer, p. 107 (2016)

9. A.A. Aleksandrov, P.A. Kapyrin, N.A. Meshkov, K.A. Neusypin, A.E. Popovich \& A.V. Proletarsky, Gamification in the advanced higher professional education: Fundamentals of theory and experience of use, International Journal of Civil Engineering and Technology, Vol. 9, I. 11, November 2018, pp. 1800-1808 (2018). Article ID: IJCIET_09_11_176

10. A.V. Proletarsky and K.A. Neusypin, Particularities of using the modern information technologies in education, European Social Science Journal, Vol. 1-1, pp. 63-65 (2014)

11. V.N. Zimin, S.A. Mardanov and D.A. Sergeev, Theoretical and practical bases of shaping the professional paths for students of IT specialties, International Journal of Experimental Education, Vol. 8, pp. 34-38 (2017)

12. L.D. Feisel \& A.J. Rosa, The Role of Laboratory in Engineering Education, Journal Engineering Education, Vol. 94, No. 1, pp. 121-130 (2005)

13. R.W. Lucky, The future of engineering, IEEE Spectrum, Vol. 35, p. 86 (2002)

14. T.I. Buldakova \& A.Sh. Dzhalolov, Analysis of data processes and choices of dataprocessing and security technologies in situation centers, Scientific and Technical Information Processing 39(2), pp. 127-132 (2012). DOI: 10.3103/S0147688212020116

15. R. Paradiso, G. Loriga \& N. Taccini, A wearable health care system based on knitted integrated sensors, IEEE Transactions on Information Technology in Biomedicine, Vol. 9, No. 3, pp. 337-344 (2005)

16. J. Winters \& Y. Wang, Wearable sensors and telerehabilitation, IEEE Engineering in Medicine and Biology Magazine, Vol. 22, No. 3, pp. 56-65 (2003) 
17. A. Biswas \& P. Sarthak, Smart Collar Short range signal triangulation for animal monitoring, International Journal of Advanced Research, Vol. 4, No. 1, pp. 1528- 1535 (2016)

18. T.Yu. Tsibizova and V.N. Zimin, Development of a way for fulfilling the function of habilitation of students and graduates of educational organizations in the present-day conditions, Automation, Modern Technologies, Vol. 71(10), pp. 465-468 (2017)

19. V.N. Zimin, S.A. Mardanov and D.A. Sergeev, Theoretical and practical bases of shaping the professional paths for students of IT specialties, International Journal of Experimental Education, Vol. 8, pp. 34-38 (2017)

20. T. Buldakova, D. Krivosheeva, Data Protection During Remote Monitoring of Person's State, Dolinina O at al (eds) Recent Research in Control Engineering and Decision Making, ICIT-2019. Studies in Systems, Decision and Control, Vol. 199, Springer, Cham, pp. 3-14 (2019). https://doi.org/10.1007/978-3-030-12072-6_1.

21. T.I. Buldakova, Cybersecurity Risks Analyses at Remote Monitoring of Object's State, Kravets A., Bolshakov A., Shcherbakov M. (eds) Cyber-Physical Systems: Industry 4.0 Challenges, Studies in Systems, Decision and Control, Vol. 260, Springer, Cham (2020). DOI: 10.1007/978-3-030-32648-7_15.

22. S.I. Suyatinov, Conceptual Approach to Building a Digital Twin of the Production System, Kravets A., Bolshakov A., Shcherbakov M. (eds) Cyber-Physical Systems: Industry 4.0 Challenges, Studies in Systems, Decision and Control, Vol. 259, Springer, Cham (2020). DOI: 10.1007/978-3-030-32579-4_22.

23. J. Herwan, S. Kano, O. Ryabov, H. Sawada \& N. Kasashima, Cyber-physical system architecture for machining production line, 2018 IEEE Industrial Cyber-Physical Systems (ICPS), pp 387-391 (2018). https://doi.org/10.1109/ICPHYS.2018.8387689.

24. O. Sénéchal \& D. Trentesaux, A framework to help decision makers to be environmentally aware during the maintenance of cyber physical systems, Environmental Impact Assessment Review, 77, pp. 11-22 (2019). http://dx.doi.org/10.1016/j.eiar.2019.02.007.

25. S. Chernyshev, Cyber-Physical Principles of Information Processing in UltraWideband Systems, Kravets A., Bolshakov A., Shcherbakov M. (eds), Cyber-Physical Systems: Industry 4.0 Challenges. Studies in Systems, Decision and Control, Vol. 260, Springer, Cham (2020). DOI: 10.1007/978-3-030-32648-7_1.

26. S.I. Suyatinov, Criteria and Method for Assessing the Functional State of a Human Operator in a Complex Organizational and Technical System, Global Smart Industry Conference (GloSIC), Chelyabinsk, Russia, pp. 1-6 (2018). http://dx.doi.org/10.1109/GloSIC.2018.8570088.

27. M. Kedadouche, M. Thomas, A. Tahan \& R. Guilbault, Nonlinear Parameters for Monitoring Gear: Comparison Between Lempel-Ziv, Approximate Entropy, and Sample Entropy, Complexity Shock and Vibration, article ID 959380, pp. 1-12 (2015). http://dx.doi.org/10.1155/2015/959380

28. F. Isik, An Entropy-Based Approach for Measuring Complexity in Supply Chains, International Journal of Production Research 48(12), pp. 3681-3696 (2010)

29. T.I. Buldakova and S.I. Suyatinov, Assessment of the State of Production System Components for Digital Twins Technology, Kravets A., Bolshakov A., Shcherbakov M. (eds) Cyber-Physical Systems: Industry 4.0 Challenges, Systems, Decision and Control, Vol. 259 (2020). Springer, Cham. DOI: 10.1007/978-3-030-32579-4_20

30. S.M. Pincus, Approximate entropy (ApEn) as complexity measure, Chaos, Vol. 5, No. 1, pp. 110-117 (1995) 